\title{
Relativistic String Model in a Space-Time of a Constant Curvature
}

\author{
B. M. Barbashov and V. V. Nesterenko \\ Laboratory of Theoretical Physics, Joint Institute for Nuclear Research, SU-141980 Dubna, USSR
}

\begin{abstract}
The relativistic string model is investigated in a space-time of a constant curvature (de Sitter universe). The fundamental differential quadratic forms of the world surface of the string are considered as the dynamical variables. The coefficients of these forms obey two nonlinear equations

$$
\varphi_{, 11}-\varphi_{, 22}=e^{\varphi} \cos \theta+K e^{-\varphi}, \theta_{, 11}-\theta_{, 22}=e^{\varphi} \sin \theta \text {. }
$$
\end{abstract}

The Lax representation for this system is obtained.

\section{Introduction}

Relativistic string model has rather a long history (see e.g. [1-3]). In the elementary particle physics the relativistic string was introduced as the dynamical basis of the dual resonance models. In recent years the ideas of the string model are used by the investigation of the mechanism of the quark confinement in hadrons $[4,5]$ and by the representation of the Yang-Mills field theory in terms of the functionals defined on contours [6-9].

In all papers devoted to the relativistic string model the flat space-time was considered. We shall investigate this model in a space-time of a constant curvature (de Sitter universe). If we take the viewpoint that the gravitation may play an important role in the world of the elementary particles (see e.g. [10]), then the aim of this paper will not be percieved as the abstract pure mathematical problem.

We shall use the differential geometry methods when the world surface of the string is described by the differential quadratic forms rather than by the string coordinates [11-15]. In this approach the string dynamics is defined by a system of two non-linear equations. The differential geometry technique enables us to construct the Lax representation for this system (more precisely, the so-called "Zero-curvature equation" [16]).

\section{Minimal World Surfaces in de Sitter Space-Time}

The Nambu-Goto action of the relativistic string [1-3] can be easily generalized to the curved space-time [17]

$$
S=-\kappa \iint d^{2} u \sqrt{-\operatorname{det}\left\|\frac{\partial x^{\mu}}{\partial u^{i}} \frac{\partial x^{v}}{\partial u^{j}} g_{\mu v}(x)\right\|}=-\kappa \iint d^{2} u \sqrt{-\operatorname{det}\left\|a_{i j}\right\|},
$$


where $x^{\mu}\left(u^{1}=\tau, u^{2}=\sigma\right)$ is the parametric representation of the string world surface, $g_{\mu \nu}(x)$ is the metric tensor of the curved space-time, $a_{i j}=x_{, i}^{\mu} x_{, j}^{v} g_{\mu \nu}$ is the metric tensor on the string world surface, $x_{, i}^{\mu}=\partial x^{\mu} / \partial u^{i}$, and $\kappa$ is a constant with the dimension of the inverse squared length. The principle of least action, as applied to the functional (1), leads to the problem of determining two-dimensional minimal surface in the four-dimensional Riemannian space with metric $g_{\mu \nu}(x)$. The Euler equation has the form [18]

$$
\sqrt{-\bar{a}} g_{\mu \nu}\left(\square x^{\nu}+\Gamma_{\alpha \beta}^{\nu}(g) a^{i j} x_{, i}^{\alpha} x_{, j}^{\beta}\right)=0,
$$

where $\bar{a}=\operatorname{det}\left\|a_{i j}\right\|, \square x^{v}=\frac{1}{\sqrt{-\bar{a}}} \frac{\partial}{\partial u^{i}}\left(\sqrt{-\bar{a}} a^{i k} x_{, k}^{v}\right)$ is the covariant LaplaceBeltrami operator with respect to the inner metric on the string world surface $a_{i j}$, $\Gamma_{\alpha \beta}^{v}(g)$ are the Christoffel symbols for the metric tensor $g_{\mu \nu}(x)$. If the space-time is flat, $\Gamma_{\alpha \beta}^{\nu}(g)=0$, and we obtain the well known result [19] in the theory of the minimal varieties in Euclidean space $\square x^{v}=0$.

Further we suppose that the space-time has a constant curvature (de Sitter universe). In terms of the Weierstrass coordinates $z^{\mu}, \mu=1,2, \ldots, 5$ de Sitter universe can be represented as the hyperboloid in the five-dimensional pseudoEuclidean space [20]

$$
\left(z^{1}\right)^{2}-\left(z^{2}\right)^{2}-\left(z^{3}\right)^{2}-\left(z^{4}\right)^{2}+\varepsilon\left(z^{5}\right)^{2}=\varepsilon R^{2},
$$

where $\varepsilon=+1$ for the de Sitter space-time of the first kind and $\varepsilon=-1$ for the de Sitter space-time of the second kind.

Instead of solving equation (2) for the string coordinates $x^{\mu}\left(u^{1}, u^{2}\right)$ with a given space-time metric $g_{\mu v}(x)$ we shall use the differential geometry technique [18] by which the string world surface can be described by its fundamental quadratic forms $a_{i j}\left(u^{1}, u^{2}\right), b_{\alpha \mid i j}\left(u^{1}, u^{2}\right)$ and torsion vectors $v_{\alpha \beta \mid i}\left(=-v_{\beta \alpha \mid i}\right), i, j=1,2 ; \alpha, \beta=3,4$. The thebrem on the embedding of the Riemannian manifolds tells that these quantities will define a two-dimensional surface embedded in space-time of a constant curvature up to its motion as a whole if and only if the equations of Gauss

$$
R_{i j k l}=\sum_{\sigma=3}^{4} e_{\sigma}\left(b_{\sigma \mid i k} b_{\sigma \mid j l}-b_{\sigma \mid l i} b_{\sigma \mid j k}\right)+\frac{\varepsilon}{R^{2}}\left(a_{i k} a_{j l}-a_{i l} a_{j k}\right),
$$

Codazzi

$$
b_{\alpha \mid j i j ; k}-b_{\alpha \mid i k ; j}=\sum_{\beta=3}^{4} e_{\beta}\left(v_{\beta \alpha \mid k} b_{\beta \mid i j}-v_{\beta \alpha \mid j} b_{\beta \mid i k}\right),
$$

and Ricci

$$
\begin{gathered}
v_{\beta \alpha \mid j ; k}-v_{\beta \alpha \mid k ; j}+\sum_{\gamma=3}^{4} e_{\gamma}\left(v_{\gamma \beta \mid j} v_{\gamma \alpha \mid k}-v_{\gamma \beta \mid k} v_{\gamma \alpha \mid j}\right) \\
+g^{l m}\left(b_{\beta \mid l j} b_{\alpha \mid m k}-b_{\beta \mid k} b_{\alpha \mid m j}\right)=0, e_{\alpha}= \pm 1
\end{gathered}
$$

will be satisfied. In these equations the Latin indices take values 1, 2 and the Greek indices 3,4 . The left-hand side of Eq. (4), $R_{i j k l}$, is the Riemann curvature tensor 
defined by the metric tensor of the world surface of the string $a_{i j}$ [18]. The semicolon in Eqs. (4)-(6) means the covariant differentiation with respect to the metric tensor $a_{i j}$.

The metric of space-time $g_{\mu v}(x)$ does not enter into Eqs. (4)-(6) explicitly. The only consequence of the constant curvature of space-time is the second term in the right-hand side of the Gauss equation (4).

From the equation of motion (2) it follows that the world sheet of the string has to be a minimal surface [18]

$$
a^{i j} b_{\alpha \mid i j}=0, \quad \alpha=3,4 .
$$

On the string world surface the isometric coordinates can be chosen

$$
a_{11}=-a_{22}=a, a_{12}=0 .
$$

In this coordinate system conditions (7) take the form

$$
b_{\alpha \mid 11}=b_{\alpha \mid 22}, \quad \alpha=3,4 .
$$

Eliminating from the Codazzi equation (6) the torsion vector $v_{i}=\left\{v_{1}=v_{43 \mid 1}\right.$, $\left.v_{2}=v_{43 \mid 2}\right\}$ and taking into account (9) we obtain

$$
\frac{\partial}{\partial u^{\mp}} \sum_{\alpha=3}^{4}\left(b_{\alpha \mid 11} \pm b_{\alpha \mid 12}\right)^{2}=0
$$

with $u^{ \pm}=u^{1} \pm u^{2}$. Therefore

$$
\sum_{\alpha=3}^{4}\left(b_{\alpha \mid 11} \pm b_{\alpha \mid 12}\right)^{2}=q_{ \pm}\left(u^{ \pm}\right)
$$

where $q_{ \pm}$are two arbitrary functions. As in the case of the flat space-time [21] it is convenient to introduce the following variables

$$
\begin{gathered}
b_{3 \mid 11} \pm b_{3 \mid 12}=q_{ \pm}\left(u^{ \pm}\right) \cos \alpha_{ \pm}, \\
b_{4 \mid 11} \pm b_{4 \mid 12}=q_{ \pm}\left(u^{ \pm}\right) \sin \alpha_{ \pm}, \\
\alpha_{+}-\alpha_{-}=\theta, \quad a_{11}=-a_{22}=a=e^{\varphi} .
\end{gathered}
$$

In terms of the new variables the Gauss equation (4) and the Ricci equation (6) part from the system (4)-(6)

$$
\begin{aligned}
\varphi_{, 11}-\varphi_{, 22} & =2 q_{+}\left(u^{+}\right) q_{-}\left(u^{-}\right) e^{\varphi} \cos \theta+2 K e^{-\varphi}, \\
\theta_{, 11}-\theta_{, 22} & =2 q_{+}\left(u^{+}\right) q_{-}\left(u^{-}\right) e^{\varphi} \sin \theta, K=\varepsilon / R^{2},
\end{aligned}
$$

but the Codazzi equations (5) take the form

$$
v_{1}+v_{2}=\alpha_{-, 1}+\alpha_{-, 2}, \quad v_{1}-v_{2}=\alpha_{+, 1}-\alpha_{+, 2} .
$$

The arbitrary functions $q_{ \pm}\left(u^{ \pm}\right)$can be eliminated from Eq. (10) by the following change of variables

$$
\begin{gathered}
\tilde{u}^{ \pm}=\int d u^{ \pm} \sqrt{2 q_{ \pm}\left(u^{ \pm}\right)}, \sqrt{q_{+}\left(u^{+}\right) q_{-}\left(u^{-}\right)} e^{\varphi\left(u^{1}, u^{2}\right)}=e^{\tilde{\varphi}\left(\tilde{u}^{1}, \tilde{u}^{2}\right)}, \\
\theta\left(u^{1}, u^{2}\right)=\tilde{\theta}\left(\tilde{u}^{1}, \tilde{u}^{2}\right) .
\end{gathered}
$$


Now Eq. (10) become

$$
\begin{aligned}
\varphi_{, 11}-\varphi_{, 22} & =e^{\varphi} \cos \theta+K e^{-\varphi}, \\
\theta_{, 11}-\theta_{, 22} & =e^{\varphi} \sin \theta,
\end{aligned}
$$

in which the mark $\sim$ is omitted for the notation simplicity. Thus the equations of motion which completely determine, in the given approach, the relativistic string dynamics in de Sitter space-time are Eq. (12). These equations have to be complemented with the boundary conditions if the relativistic string is of finite extension. For example, for a closed string, $0 \leqq u^{2} \leqq \pi$, we have

$$
\varphi\left(u^{1}, 0\right)=\varphi\left(u^{1}, \pi\right), \quad \theta\left(u^{1}, 0\right)=\theta\left(u^{1}, \pi\right) .
$$

The change of variables

$$
\begin{gathered}
\tilde{u}^{ \pm}=|K|^{1 / 4} u^{ \pm}, \exp \varphi\left(u^{1}, u^{2}\right)=|K|^{1 / 2} \exp \tilde{\varphi}\left(\tilde{u}^{1}, \tilde{u}^{2}\right), \\
\theta\left(u^{1}, u^{2}\right)=\tilde{\theta}\left(\tilde{u}^{1}, \tilde{u}^{2}\right), \quad K \neq 0
\end{gathered}
$$

enables us to remove the constant $K$ from Eq. (12)

$$
\begin{aligned}
\varphi_{, 11}-\varphi_{, 22} & =e^{\varphi} \cos \theta+\varepsilon \mathrm{e}^{-\varphi}, \\
\theta_{, 11}-\theta_{, 22} & =e^{\varphi} \sin \theta, \quad \varepsilon= \pm 1 .
\end{aligned}
$$

\section{Lax Representation for System (13)}

In differential geometry [18] the Gauss-Codazzi-Ricci equations (4)-(6) are derived as the compatibility conditions of two systems of partial differential equations of the first order which describe the moving frame on the string world surface. Therefore, these equations can be taken as a pair of the Lax operators required for solving the nonlinear equations (13) by the inverse scattering method $[22,23]$. In de Sitter space-time we introduce the Weierstrass coordinates (3) and as the moving frame we take two tangent vectors $z_{, 1}^{\mu}, z_{, 2}^{\mu}$, two unit normals $\eta_{3}^{\mu}, \eta_{4}^{\mu}$, and vector $z^{\mu}$. In the theory of relativistic string [1-3] vector $z_{, 1}^{\mu}$ has to be time-like and vectors $z_{, 2}^{\mu}, \eta_{3}^{\mu}, \eta_{4}^{\mu}$ space-like. With this fact and the choice of signs in the quadratic form (3), we obtain the following equations [18]

$$
\begin{aligned}
& z_{; i j}^{\mu}=-\sum_{\sigma=3}^{4} b_{\sigma \mid i j} \eta_{\sigma}^{\mu}-K a_{i j} z^{\mu}, \\
& \eta_{\sigma, i}^{\mu}=-b_{\sigma \mid i j} a^{j m} z_{, m}^{\mu}-\sum_{\tau} v_{\tau \sigma \mid i} \eta_{\tau}^{\mu},
\end{aligned}
$$

which describe the motion of the basis

$$
z_{, 1}^{\mu}, z_{, 2}^{\mu}, \eta_{3}^{\mu}, \eta_{4}^{\mu}, z^{\mu}
$$

on the string world surface. The contraction with $a^{i j}$ of the first equation in (14), by virtue of (7), gives the known result [24] in the theory of minimal varieties embedded in a space of a constant curvature, namely, the action of the LaplaceBeltrami operator on vector $z^{\mu}$ is

$$
\square z^{\mu}=-2 K z^{\mu} .
$$


Instead of vectors (15) we introduce the orthonormal moving basis

$$
e_{1}^{\mu}=\frac{z_{, 1}^{\mu}}{\sqrt{a}}, e_{2}^{\mu}=i \frac{z_{, 2}^{\mu}}{\sqrt{a}}, e_{3}^{\mu}=i \eta_{3}^{\mu}, e_{4}^{\mu}=i \eta_{4}^{\mu}, e_{5}^{\mu}=\sqrt{\varepsilon} \frac{z^{\mu}}{R} .
$$

Using (14) we obtain the following equations describing the motion of the basis (16) on the string world surface

$$
\frac{\partial e_{a}}{\partial u^{j}}=\sum_{b=1}^{5} \omega_{a b}^{j} e_{b}, \quad a, b=1,2, \ldots, 5 ; i, j=1,2,
$$

where

$$
\omega_{a b}^{1}=\left|\begin{array}{ccccc}
0 & -i \frac{a_{22}}{2 a} & i \frac{b_{3 \mid 11}}{\sqrt{a}} & i \frac{b_{4 \mid 11}}{\sqrt{a}} & -\frac{\sqrt{\varepsilon a}}{R} \\
i \frac{a_{, 2}}{2 a} & 0 & -\frac{b_{3 \mid 12}}{\sqrt{a}} & -\frac{b_{4 \mid 12}}{\sqrt{a}} & 0 \\
-i \frac{b_{3 \mid 11}}{\sqrt{a}} & \frac{b_{3 \mid 12}}{\sqrt{a}} & 0 & -v_{1} & 0 \\
-i \frac{b_{4 \mid 11}}{\sqrt{a}} & \frac{b_{4 \mid 12}}{\sqrt{a}} & v_{1} & 0 & 0 \\
\frac{\sqrt{\varepsilon a}}{R} & 0 & 0 & 0 & 0 \\
0 & -i \frac{a, 1}{2 a} & i \frac{b_{3 \mid 12}}{\sqrt{a}} & \frac{b_{4 \mid 12}}{\sqrt{a}} & 0 \\
i \frac{a, 1}{2 a} & 0 & -\frac{b_{3 \mid 22}}{\sqrt{a}} & -\frac{b_{4 \mid 22}}{\sqrt{a}} & i \frac{\sqrt{\varepsilon a}}{R} \\
-i \frac{b_{3 \mid 12}}{\sqrt{a}} & \frac{b_{3 \mid 22}}{\sqrt{a}} & 0 & -v_{2} & 0 \\
-i \frac{b_{4 \mid 12}}{\sqrt{a}} & \frac{b_{4 \mid 22}}{\sqrt{a}} & v_{2} & 0 & 0 \\
0 & -i \frac{\sqrt{\varepsilon a}}{R} & 0 & 0 & 0
\end{array}\right|,
$$

We can reduce the dimension of the matrix equations (17) in the following way. The skewsymmetric matrices (18) and (19) describe infinitesimal rotations in the five-dimensional pseudo-Euclidean space. Some matrix elements in (18) and (19) are imaginary because the quadratic form (3) is indefinite. The matrix representation of the minimal dimension of the $O(5)$-group can be constructed in terms of the usual $(4 \times 4)$ Dirac $\gamma$-matrices [25]. In this representation the $O(5)$-generators are

$$
\begin{gathered}
I_{\mu \nu}=\frac{1}{4}\left[\gamma_{\mu}, \gamma_{\nu}\right], \\
\gamma_{\mu} \gamma_{\nu}+\gamma_{\nu} \gamma_{\mu}=2 \delta_{\mu \nu}, \gamma_{\mu}^{+}=\gamma_{\nu}, \quad \mu, \nu=1,2, \ldots, 5 .
\end{gathered}
$$


In what follows we shall use the representation of the $\gamma$-matrices in which $\gamma_{5}=\gamma_{1} \gamma_{2} \gamma_{3} \gamma_{4}$ is diagonal [26]

$$
\gamma_{k}=\left(\begin{array}{cc}
0 & i \sigma_{k} \\
-i \sigma_{k} & 0
\end{array}\right), k=1,2,3, \gamma_{4}=\left(\begin{array}{ll}
0 & I \\
I & 0
\end{array}\right), \gamma_{5}=\left(\begin{array}{cc}
-I & 0 \\
0 & I
\end{array}\right),
$$

where $\sigma_{k}$ are the Pauli matrices,

Now we can correlate to Eq. (17) two systems of partial differential equations each containing only four equations

$$
\frac{\partial \psi_{a}}{\partial u^{j}}=\frac{1}{2} \sum_{\mu, v>1} \omega_{\mu v}^{j}\left(\gamma_{\mu} \gamma_{v}\right)_{a b} \psi_{b}=\frac{1}{2} \Omega_{a b}^{j} \psi_{b}, \quad j=1,2,
$$

where $\psi=\psi\left(u^{1}, u^{2}\right)$ is a four-component function and $4 \times 4$ matrices $\Omega^{j}$ are

$$
\Omega^{j}=\sum_{\mu>v=1}^{5} \omega_{\mu \nu}^{j} \gamma_{\mu} \gamma_{\nu}, \quad j=1,2 .
$$

To write out these matrices explicitly we introduce the notation

$$
\Omega^{j}=\left(\begin{array}{ll}
a_{11}^{j} & a_{12}^{j} \\
a_{21}^{j} & a_{22}^{j}
\end{array}\right)
$$

where $a_{k l}^{j}$ are again the $(2 \times 2)$ matrices. Using Eqs. (18)-(20) and (22) we obtain the following expansions of $a_{k l}^{j}, j, k, l=1,2$ in terms of the Pauli matrices $\sigma_{k}$

$$
\begin{gathered}
a_{11}^{1}=-i \sigma_{1}\left(Q_{+}^{*}-Q_{-}\right)+\sigma_{2}\left(Q_{+}^{*}+Q_{-}\right)-\frac{\sigma_{3}}{4}\left(\varphi_{, 2}+2 i v_{1}\right), \\
a_{12}^{1}=a_{21}^{1}=-i \sigma_{1} \frac{\sqrt{\varepsilon}}{R} e^{-\varphi / 2}, \\
a_{22}^{1}=-i \sigma_{1}\left(Q_{+}-Q_{-}^{*}\right)+\sigma_{2}\left(Q_{+}+Q_{-}^{*}\right)-\frac{\sigma_{3}}{4}\left(\varphi_{, 2}-2 i v_{1}\right), \\
a_{11}^{2}=-i \sigma_{1}\left(Q_{+}^{*}+Q_{-}\right)+\sigma_{2}\left(Q_{+}^{*}-Q_{-}\right)-\frac{\sigma_{3}}{4}\left(\varphi_{, 1}+2 i v_{2}\right), \\
a_{12}^{2}=a_{21}^{2}=-\sigma_{2} \frac{\sqrt{\varepsilon}}{2 R} e^{-\varphi / 2}, \\
a_{22}^{2}=-i \sigma_{1}\left(Q_{+}+Q_{-}^{*}\right)+\sigma_{2}\left(Q_{+}-Q_{-}^{*}\right)-\frac{\sigma_{3}}{4}\left(\varphi_{, 1}-2 i v_{2}\right),
\end{gathered}
$$

in which

$$
Q_{ \pm}=\frac{1}{4} \exp \left(\frac{\varphi}{2} \pm i \alpha_{ \pm}\right), \quad \theta=\alpha_{+}-\alpha_{-}, \varepsilon= \pm 1
$$

and $v_{i}, i=1,2$ is connected with $\alpha_{ \pm}$by formulae (11). 
The compatibility conditions of the linear equations (21)

$$
\Omega_{, 2}^{1}-\Omega_{, 1}^{2}=\left[\Omega^{2}, \Omega^{1}\right]
$$

results in Eq. (13).

The transition to new variables $\bar{u}^{1}, \bar{u}^{2}$

$$
\bar{u}^{1}+\bar{u}^{2}=\lambda\left(u^{1}+u^{2}\right), \quad \bar{u}^{1}-\bar{u}^{2}=\lambda^{-1}\left(u^{1}-u^{2}\right)
$$

enables us to introduce the spectral parameter $\lambda$ into Eq. (21). This change of variables retains the form of system (13), while the matrix elements $\Omega_{a b}^{j}$ (23) obtain factors $(\lambda \pm 1 / \lambda)$ (see e.g. $[27,28]$ ). We shall not perform here these simple transformations.

\section{Conclusion}

The proposed model of the relativistic string in a space-time of a constant curvature gives us one more example of a system of two nonlinear equations integrable by the inverse scattering technique. This system was derived in paper [29] by investigating the world surfaces with a constant mean curvature in the four-dimensional Minkowski space-time. In the same paper the Lax representation for this system was obtained in terms of $2 \times 2$ matrices. Therefore, it would be interesting to reduce the dimension of matrices $\Omega^{j}$ in the linear spectral problem (21).

\section{References}

1. Rebbi, G.: Phys. Rep. 12C, 1-73 (1974)

2. Scherk, J.: Rev. Mod. Phys. 47, 123-164 (1975)

3. Barbashov, B.M., Nesterenko, V.V.: Phys. Elem. Part. Atom. Nucl. (in Russian) 9, 709-758 (1978)

4. Nambu, Y.: Phys. Rev. D10, 4262-4268 (1974); Phys. Rep. 23C, 250-253 (1976)

5. Wilson, K.G.: Phys. Rev. D 10, 2445-2459 (1974); Phys. Rep. 23C, 331-347 (1976)

6. Nambu, Y.: Phys. Lett. 80 B, 372-377 (1979)

7. Gervais, J.L., Neveu, A. : Phys. Lett. 80 B, 255-258 (1979)

8. Polyakov, A.M.: Phys. Lett. 82B, 247-250 (1979)

9. Durand, L., Mendel, E.: Phys. Lett. 85B, 241-245 (1979)

10. Markov, M.A.: Can the gravitational field prove essential for the theory of elementary particles? Prog. Theor. Phys., Suppl. Extra Number, 85-95 (1965)

11. Lund, F., Regge, T.: Phys. Rev. D14, 1524-1535 (1976)

12. Lund, F.: Phys. Rev. D15, 1540 (1977)

13. Lund, F.: Phys. Rev. Lett. 38, 1175-1178 (1977)

14. Omnes, R.: Nucl. Phys. B149, 269-284 (1979)

15. Barbashov, B.M., Nesterenko, V.V., Chervjakov, A.M.: Lett. Math. Phys. 3, 359-365 (1979)

16. Faddeev, L.D., Korepin, V.E.: Phys. Rep. 42C, 1-87 (1978)

17. Ademolo, M. et al. : Nuovo Chim. 21A, 77-145 (1974)

18. Eisenhart, L.P.: Riemannian geometry. Princeton: Princeton University Press 1949

19. Osserman, R.: Bull. Am. Math. Soc. 75, 1092-1120 (1969)

20. Hawking, S.W., Ellis, G.F.R.: The large scale structure of space-time, pp. 124-134. Cambridge: Cambridge University Press 1973

21. Barbashov, B.M., Nesterenko, V.V., Chervjakov, A.M.: Teor. Mat. Fiz. 40, 15-27 (1979)

22. Lax, P.D.: Commun. Pure Appl. Math. 21, 467 (1968)

23. Ablowitz, M.J., Kaup, D.J., Newell, A.C., Segur, H. : Phys. Rev. Lett. 30, 1262-1264 (1973); Stud. Appl. Math. 53, 249 (1974) 
24. Takahashi, T.: J. Math. Soc. Jpn. 18, 380-386'(1966)

25. Gursey, F.: Introduction to group theory. Article in: Relativity, groups, and topology (eds. DeWitt, C., DeWitt, B.), pp. 89-161. New York, London: Gordon and Breach 1964

26. Pauli, W.: Wellenmechanik, Zurich, 1959

27. Pohlmeyer, K.: Commun. Math. Phys. 46, 209-221 (1976)

28. Neveu, A., Papanicolaou, N.: Commun. Math. Phys. 58, 31-63 (1978)

29. Barbashov, B.M., Nesterenko, V.V., Chervjakov, A.M.: On the theory of world surfaces of a constant mean curvature. Preprint JINR P2-12946, Dubna 1979

Communicated by $\mathrm{H}$. Araki

Received April 24, 1980; in revised form July 21, 1980 